\title{
Sampled-Data Synchronization for Complex Dynamical Networks with Time-Varying Coupling Delay and Random Coupling Strengths
}

\author{
Jian-An Wang and Xin-Yu Wen \\ School of Electronics Information Engineering, Taiyuan University of Science and Technology, Taiyuan, Shanxi 030024, China \\ Correspondence should be addressed to Jian-An Wang; wangjianan588@163.com
}

Received 14 January 2014; Revised 31 March 2014; Accepted 14 April 2014; Published 6 May 2014

Academic Editor: He Huang

Copyright (C) 2014 J.-A. Wang and X.-Y. Wen. This is an open access article distributed under the Creative Commons Attribution License, which permits unrestricted use, distribution, and reproduction in any medium, provided the original work is properly cited.

\begin{abstract}
This paper is concerned with the problem of sampled-data synchronization for complex dynamical networks (CDNs) with timevarying coupling delay and random coupling strengths. The random coupling strengths are described by normal distribution. The sampling period considered here is assumed to be less than a given bound. By taking the characteristic of sampled-data system into account, a discontinuous Lyapunov functional is constructed, and a delay-dependent mean square synchronization criterion is derived. Based on the proposed condition, a set of desired sampled-data controllers are designed in terms of linear matrix inequalities (LMIs) that can be solved effectively by using MATLAB LMI Toolbox. Numerical examples are given to demonstrate the effectiveness of the proposed scheme.
\end{abstract}

\section{Introduction}

In the real world, many practical and natural systems can be described by models of complex networks such as internet, food webs, electric power grids, scientific citation networks, and social networks. Therefore, a dynamical network can be regarded as a dynamical system with a special structure. In the last few years, complex dynamical networks (CDNs) have received extensive attention and increasing interest across many fields of science and engineering [1-3]. CDNs are a large set of interconnected nodes, in which each node represents an element with certain dynamical system and edge represents the relationship between them. With the important discovery of the "small-world" and "scale-free" properties, complex dynamical networks have become a focal research topic in the area of complexity science.

It is very common that many natural systems often exhibit collective cooperative behaviors among their constituents. Synchronization, as a kind of typical collective behavior, is one of key issues in the study of complex dynamical networks. The main reason is that network synchronization not only can explain many natural phenomena but also has wide applications in many fields including secure communications, synchronous information exchange in the internet, genetic regulatory process, the synchronous transfer of digital signals in communication networks, and so on. Over the past several decades, the synchronization in CDNs has been intensively investigated from various fields such as sociology, biology, and physics [4-16]. The authors in [5] focused the synchronization stability of general CDNs with coupling delay. In [6], the authors investigated the locally and globally adaptive synchronization of an uncertain complex dynamical network. The problem of globally exponential synchronization of impulsive dynamical networks was investigated in [7]. The pinning synchronization problems in CDNs have been analyzed in $[8,9]$. In [10], the authors studied the global exponential synchronization and synchronizability for general dynamical networks. In [11], some sufficient conditions for CDNs with and without coupling delays in the state to be passive were presented. Recently, the guaranteed cost synchronization of a CDN via dynamic feedback control was addressed in [15].

It is well known that the coupling strength of complex dynamical network plays an important role in the realizing 
synchronization. In general, the coupling strength of the considered CDNs is deterministic [4-16]. If the deterministic coupling strength is large enough, a complex network can realize synchronization by itself. However, according to the discussion in $[17,18]$, because of the effects of environment and artificial factor, the coupling strength of complex dynamical networks may randomly vary around some constants. If the upper or lower bound of the random coupling strength is only considered, some conservative result will be derived. That is to say, random phenomena in coupling strength should be taken into account when dealing with the synchronization of CDNs. Furthermore, the normal distribution characteristic of random variables can be easily obtained by statistical methods. Therefore, it is interesting to investigate the synchronization of CDNs with random coupling strengths described by normal distribution.

On the other hand, the sampled-data control system, whose control signals are allowed to change only at discrete sampling instants, can drastically reduce the amount of information transmitted and increase the efficiency of bandwidth usage. Therefore, sampled-data control has received notable attention [19-22]. The input delay approach proposed in [19] is very popular in the study of sampled control system, where the system is modeled as a continuous-time system with a time-varying sawtooth delay in the control input induced by sample-and-hold. In [20], by constructing the time-dependent Lyapunov functional, a refined input delay approach was presented. Later, the chaos synchronization problems are investigated by using sampled-data control [23-26]. Recently, in the framework of the input delay approach, the sampled-data synchronization problem has been investigated for a class of general complex networks with time-varying coupling delays in [27]. Furthermore, some improved sampled-data synchronization criterion has been derived to ensure the exponential stability of the closed-loop error system and corresponding sampled-data feedback controllers are designed in [28]. By combining the time-dependent Lyapunov functional approach and convex combination technique, the exponential sampled-data synchronization of CDNs with time-varying coupling delay and uncertain sampling was studied in [29]. However, the Lyapunov functional proposed in $[27,28]$ ignored the substitutive characteristic of sampled-data system, which leads to some conservatism inevitably. In addition, the results obtained in [29] are sufficient conditions, which imply that there is still room for further improvement. To the best of our knowledge, the sampled-data synchronization problem of complex dynamical networks with time-varying coupling delays and random coupling strengths has not been studied in the literature.

Motivated by the aforementioned discussions, in this paper, the problem of sampled-data synchronization of CDNs with time-varying coupling delay and random coupling strengths is investigated. The sampling period is assumed to be time varying but less than a given bound. The random coupling strengths are described by normal distribution. By capturing the characteristic of sampled-data control system, a new discontinuous Lyapunov functional is constructed. By using the low bound lemma and convex combination approach, a mean square synchronization condition is formulated in terms of LMIs. The corresponding sampled-data controllers are designed that can achieve the synchronization of the considered CDNs. The proposed method can lead to a less conservative result than the existing ones. Finally, numerical examples are given to illustrate the effectiveness and less conservatism of the presented sampled-data control scheme.

Notation. The notations used throughout this paper are fairly standard. $R^{n}$ and $R^{m \times n}$ denote the $n$-dimensional Euclidean space and the set of all $m \times n$ real matrix, respectively. $P>0$ or $P<0$ means that $P$ is symmetric and positive or negative definite. The superscript " $T$ " represents the transpose, and " $I$ " and "0" denote the identity and zero matrix with appropriate dimensions. $\operatorname{diag}\left\{l_{1}, l_{2}, \ldots, l_{n}\right\}$ stands for a block diagonal matrix. The symmetric terms in a symmetric matrix are denoted by ${ }^{*}$.

\section{Preliminaries and Model Description}

Consider a class of linearly coupled complex dynamical networks consisting of $N$ identical coupled nodes, in which each node is an $n$-dimensional subsystem

$$
\begin{array}{r}
\dot{x}_{i}(t)=f\left(x_{i}(t)\right)+c_{1}(t) \sum_{j=1}^{N} G_{i j} D x_{j}(t) \\
+c_{2}(t) \sum_{j=1}^{N} G_{i j} A x_{j}(t-\tau(t))+u_{i}(t), \\
i=1,2, \ldots, N,
\end{array}
$$

where $x_{i}=\left(x_{i 1}, x_{i 2}, \ldots, x_{i n}\right) \in R^{n}$ and $u_{i}(t) \in R^{n}$ are, respectively, the state variable and the control input of the node i.f : $R^{n} \rightarrow R^{n}$ is a continuous vectorvalued function. $c_{1}(t)$ and $c_{2}(t)$ are mutually independent random variables, which denote the random coupling strengths of nondelayed coupling and time-delayed couplings, respectively. $\tau(t)$ denotes the time-varying coupling delay satisfying $0 \leq \tau(t) \leq h, \dot{\tau}(t) \leq \mu$, where $h>$ 0 and $\mu$ are known constants. $D=\left(d_{i j}\right)_{n \times n} \in R^{n \times n}$ is the constant inner-coupling matrix and $A=\left(a_{i j}\right)_{n \times n} \in R^{n \times n}$ is the time-delay inner-coupling matrix. $G=\left(g_{i j}\right) \in R^{N \times N}$ is the coupling configuration matrix, where $g_{i j}$ is defined as follows: if there is a connection between node $i$ and node $j$ $(i \neq j)$, then $g_{i j}>0$; otherwise, $g_{i j}=0$, and the diagonal elements of matrix $G$ are defined by $g_{i i}=-\sum_{j=1, j \neq i}^{N} G_{i j}, i=$ $1,2, \ldots, N$

Remark 1. The coupling configuration matrix $G$ represents the topological structure of network (1). In this paper, the matrix $G$ is not assumed to be symmetric or irreducible. In $[27,28]$, the coupling configuration matrix is assumed to be symmetric, which is quite restrictive in practice. In this regards, the network model considered here is more general. 
In this paper, similar to $[17,18]$, we assume that almost all the values of $c_{i}(t), i=1,2$, are taken on some nonnegative intervals, that is, $c_{i}(t) \in\left(\sigma_{i}, \rho_{i}\right)$, where $\sigma_{i}, \rho_{i}(i=1,2)$ are nonnegative constants with $\sigma_{i}<\rho_{i}$. Almost all the values of $c_{i}(t)$ satisfying $c_{i}(t) \in\left(\sigma_{i}, \rho_{i}\right)$ imply that $\operatorname{Prob}\left\{c_{i}(t) \in\right.$ $\left.\left(\sigma_{i}, \rho_{i}\right)\right\}=1$. It should be noted that the actual minimum and maximum allowable coupling strength bounds are not $\sigma_{i}$ and $\rho_{i}$, respectively. It just means that $\operatorname{Prob}\left\{c_{i}(t)<\right.$ $\left.\sigma_{i}\right\}=0$ and $\operatorname{Prob}\left\{c_{i}(t)>\rho_{i}\right\}=0$. The actual lower bounds of $c_{i}(t)$ may be very small and the actual upper bounds of them may be very large. This is very different from synchronization results obtained by traditional method, in which constant coupling strength is always preassumed or deterministic.

Remark 2. We assume the coupling strengths satisfy the normal distribution, which can randomly vary around some given intervals. This is very different from the considered network models in [27-29], in which constant coupling strengths are always preassumed or deterministic. Therefore, for the random coupling strength, most of existing results with constant coupling strength may not be applicable anymore. In addition, it is worth pointing out that when $c_{1}(t)=$ $c_{10}$ and $c_{2}(t)=c_{20}$ or $\delta_{1}=\delta_{2}=0$, system (1) includes the models in [27-29] as a special case.

Assumption 3. There nonlinear function $f$ satisfies

$$
\begin{aligned}
& {[f(x)-f(y)-U(x-y)]^{T}[f(x)-f(y)-V(x-y)]} \\
& \quad \leq 0, \quad \forall x, y \in R^{n},
\end{aligned}
$$

where $U$ and $V$ are constant matrices of appropriate dimensions.

Assumption 4. The mathematical exception and variance of $c_{i}(t)$ are $E\left\{c_{i}(t)\right\}=c_{i 0}$ and $E\left\{\left(c_{i}(t)-c_{i 0}\right)^{2}\right\}=\delta_{i}^{2}$, respectively, where $c_{i 0}$ and $\delta_{i}$ are nonnegative constants.

On the basis of the property of variables $c_{i}(t)$, system (1) can be rewritten in the following form:

$$
\begin{aligned}
\dot{x}_{i}(t)= & f\left(x_{i}(t)\right)+c_{10} \sum_{j=1}^{N} G_{i j} D x_{j}(t) \\
& +\left(c_{1}(t)-c_{10}\right) \sum_{j=1}^{N} G_{i j} D x_{j}(t) \\
& +c_{20} \sum_{j=1}^{N} G_{i j} A x_{j}(t-\tau(t)) \\
& +\left(c_{2}(t)-c_{20}\right) \sum_{j=1}^{N} G_{i j} A x_{j}(t-\tau(t))+u_{i}(t), \\
& \quad i=1,2, \ldots, N .
\end{aligned}
$$

Let $e_{i}(t)=x_{i}(t)-s(t)$ be the synchronization error, where $s(t) \in R^{n}$ is the state trajectory of the unforced isolate node $\dot{s}(t)=f(x(t))$. Then, the error dynamics is given by

$$
\begin{aligned}
\dot{e}_{i}(t)= & g\left(e_{i}(t)\right)+c_{10} \sum_{j=1}^{N} G_{i j} D e_{j}(t) \\
& +\left(c_{1}(t)-c_{10}\right) \sum_{j=1}^{N} G_{i j} D e_{j}(t) \\
& +c_{20} \sum_{j=1}^{N} G_{i j} A e_{j}(t-\tau(t)) \\
& +\left(c_{2}(t)-c_{20}\right) \sum_{j=1}^{N} G_{i j} A e_{j}(t-\tau(t))+u_{i}(t), \\
& \quad i=1,2, \ldots, N,
\end{aligned}
$$

where $g\left(e_{i}(t)\right)=f\left(x_{i}(t)\right)-f(s(t))=\left[f_{1}\left(e_{i 1}(t)\right) f_{2}\left(e_{i 2}(t)\right)\right.$ $\left.\cdots f_{n}\left(e_{i n}(t)\right)\right]^{T}$.

The control signal is assumed to be generalized by using a zero-order-hold $(\mathrm{ZOH})$ function with a sequence of hold times $0=t_{0}<t_{1}<\cdots<t_{k}<\cdots$. Therefore, the state feedback controller takes the following form:

$$
\begin{array}{r}
u_{i}=K_{i} e_{i}\left(t_{k}\right)=K_{i}\left(x_{i}\left(t_{k}\right)-s\left(t_{k}\right)\right), \quad t_{k} \leq t<t_{k+1}, \\
i=1,2, \ldots, N,
\end{array}
$$

where $K_{i}$ is the feedback gain matrix to be determined and $e_{i}\left(t_{k}\right)$ is the discrete measurement of $e_{i}(t)$ at sampling instant $t_{k}$. In this paper, the sampling is not required to be periodic, and the only assumption is that the distance between any two consecutive sampling instants is less than a given bound. It is assumed that $t_{k+1}-t_{k}=h_{k} \leq p$ for any integer $k \geq 0$, where $p>0$ represents the largest sampling interval.

By substituting (5) into (4), we obtain

$$
\begin{aligned}
\dot{e}_{i}(t)= & g\left(e_{i}(t)\right)+c_{10} \sum_{j=1}^{N} G_{i j} D e_{j}(t) \\
& +\left(c_{1}(t)-c_{10}\right) \sum_{j=1}^{N} G_{i j} D e_{j}(t) \\
& +c_{20} \sum_{j=1}^{N} G_{i j} A e_{j}(t-\tau(t)) \\
& +\left(c_{2}(t)-c_{20}\right) \sum_{j=1}^{N} G_{i j} A e_{j}(t-\tau(t))+K_{i} e_{i}\left(t_{k}\right), \\
& \quad i=1,2, \ldots, N .
\end{aligned}
$$


Furthermore, by using the Kronecker product, system (6) can be rewritten as

$$
\begin{aligned}
\dot{e}(t)= & \bar{g}(e(t))+c_{10}(G \otimes D) e(t) \\
& +\left(c_{1}(t)-c_{10}\right)(G \otimes D) e(t) \\
& +c_{20}(G \otimes A) e(t-\tau(t)) \\
& +\left(c_{2}(t)-c_{20}\right)(G \otimes A) e(t-\tau(t)) \\
& +K e\left(t_{k}\right), \quad i=1,2, \ldots, N,
\end{aligned}
$$

where $e(t)=\left[\begin{array}{llll}e_{1}^{T}(t) & e_{2}^{T}(t) & \cdots & e_{N}^{T}(t)\end{array}\right]^{T}, \bar{g}(e(t))=$ $\left[\begin{array}{llll}g^{T}\left(e_{1}(t)\right) & g^{T}\left(e_{2}(t)\right) & \cdots & g^{T}\left(e_{n}(t)\right)\end{array}\right]^{T}$, and $K=\operatorname{diag}\left\{K_{1}, K_{2}\right.$, $\left.\ldots, K_{N}\right\}$.

To proceed further, the following definition and useful lemmas are needed.

Definition 5. The coupled complex dynamical network (1) is said to be globally synchronized in mean square sense if $\lim _{t \rightarrow \infty} E\left\{\left\|e_{i}(t)\right\|^{2}\right\}=0, i=1,2, \ldots, N$, holds for any initial values.

Lemma 6 (extended Wirtinger inequality [22]). Let $z(t) \in$ $W[a, b)$ and $z(a)=0$. Then for any matrix $R>0$, the following inequality holds:

$$
\int_{a}^{b} z^{T}(\alpha) R z(\alpha) d \alpha \leq \frac{4(b-a)^{2}}{\pi^{2}} \int_{a}^{b} \dot{z}^{T}(\alpha) R \dot{z}(\alpha) d \alpha .
$$

Lemma 7 (reciprocally convex approach [30]). Let $f_{1}, f_{2}, \ldots, f_{N}: R^{m} \mapsto R$ have positive values in an open subset $D$ of $R^{m}$. Then, the reciprocally convex combination of $f_{i}$ over $D$ satisfies

$$
\min _{\left\{\alpha_{i} \mid \alpha_{i}>0, \sum_{i} \alpha_{i}=1\right\}} \sum_{i} f_{i}(t)=\sum_{i} f_{i}(t)+\max _{g_{i, j}(t)} \sum_{i \neq j} g_{i, j}(t)
$$

subject to

$$
\left\{g_{i, j}: R^{m} \longmapsto R, g_{j, i}(t) \triangleq g_{i, j}(t),\left[\begin{array}{cc}
f_{i}(t) & g_{i, j}(t) \\
g_{j, i}(t) & f_{j}(t)
\end{array}\right] \geq 0\right\} .
$$

The aim of this paper is to design a set of sampleddata controllers (5) with sampling period as big as possible to ensure synchronizing the complex network (1) in mean square sense. By some transformation, the synchronization problem of the delayed complex network (1) can be equivalently converted into the mean square asymptotical stability problem of error system (7). Therefore, we are interested in two main issues in our paper, one is to find some stability conditions for error system (7) in mean square for given $K_{i}$, and the other is to derive the design method of sampled-data controllers.

\section{Main Results}

In this section, by considering the characteristic of sampleddata system, we first give a delay-dependent condition to ensure error system (7) to be globally stable in mean square sense. Then, based on the derived condition, the design method of the sampled-data controllers is proposed. Before presenting the main results, for the sake of presentation simplicity, we denote

$$
\begin{aligned}
& \bar{U}=\frac{\left(I_{N} \otimes U\right)^{T}\left(I_{N} \otimes V\right)}{2}+\frac{\left(I_{N} \otimes V\right)^{T}\left(I_{N} \otimes U\right)}{2}, \\
& \bar{V}=-\frac{\left(I_{N} \otimes U\right)^{T}+\left(I_{N} \otimes V\right)^{T}}{2} .
\end{aligned}
$$

Theorem 8. Under Assumptions 3-4, for given controller gain matrices $K_{i}$, the error system (7) is globally asymptotically stable in mean square sense if there exist matrices $P>0, Q_{1}>$ $0, Q_{2}>0, Q_{3}>0, Z_{1}>0, Z_{2}>0, U>0, R>0, W>$ $0, S_{1}, S_{2}, N$, and a scalar $\varepsilon>0$ such that the following LMIs are satisfied:

$$
\begin{gathered}
{\left[\begin{array}{ccc}
\Sigma-p \bar{R} & \Gamma_{1} & p N \\
* & -X_{1} & 0 \\
* & * & -p U
\end{array}\right]<0,} \\
{\left[\begin{array}{cc}
\Sigma+p \bar{R} & \Gamma_{2} \\
* & -X_{2}
\end{array}\right]<0,} \\
{\left[\begin{array}{cc}
Z_{i} & S_{i} \\
* & Z_{i}
\end{array}\right] \geq 0, \quad i=1,2,}
\end{gathered}
$$

where

$$
\Sigma=\left[\begin{array}{cccccc}
\Sigma_{11} & \Sigma_{12} & \Sigma_{13} & \Sigma_{14} & \Sigma_{15} & \Sigma_{16} \\
* & \Sigma_{22} & \Sigma_{23} & -N_{4} & -N_{5} & -N_{6} \\
* & * & \Sigma_{33} & 0 & 0 & 0 \\
* & * & * & \Sigma_{44} & \Sigma_{45} & 0 \\
* & * & * & * & \Sigma_{55} & 0 \\
* & * & * & * & * & -\varepsilon I
\end{array}\right],
$$

$\Sigma_{11}=c_{10} P(G \otimes D)+c_{10}(G \otimes D)^{T} P$

$$
+Q_{1}+Q_{2}+Q_{3}-\frac{1}{4} \pi^{2} W-\varepsilon \bar{U}
$$$$
-Z_{1}-Z_{2}+N_{1}+N_{1}^{T}
$$

$\Sigma_{12}=P K+\frac{1}{4} \pi^{2} W+Z_{1}-S_{1}-N_{1}^{T}+N_{2}$,

$\Sigma_{13}=S_{1}+N_{3}$,

$\Sigma_{14}=P(G \otimes A)+Z_{2}-S_{2}+N_{4}$,

$\Sigma_{15}=S_{2}+N_{5}$,

$\Sigma_{16}=P-\varepsilon \bar{V}+N_{6}$,

$\Sigma_{22}=-2 Z_{1}-\frac{1}{4} \pi^{2} W+S_{1}+S_{1}^{T}-N_{2}-N_{2}^{T}$,

$\Sigma_{23}=Z_{1}-S_{1}-N_{3}$,

$\Sigma_{33}=-Q_{1}-Z_{1}$, 


$$
\begin{aligned}
& \Sigma_{44}=-(1-\mu) Q_{3}-2 Z_{2}+S_{2}+S_{2}^{T}, \\
& \Sigma_{45}=Z_{2}-S_{2} \text {, } \\
& \Sigma_{55}=-Q_{2}-Z_{2} \text {, } \\
& \Gamma_{1}=\left[\begin{array}{llll}
\Omega_{1}^{T} & \delta_{1} \Omega_{2}^{T} & \delta_{2} \Omega_{3}^{T}
\end{array}\right], \\
& \Gamma_{2}=\left[\begin{array}{lll}
\widehat{\Omega}_{1}^{T} & \delta_{1} \widehat{\Omega}_{2}^{T} & \delta_{2} \widehat{\Omega}_{3}^{T}
\end{array}\right] \\
& \Omega_{1}=\left[\begin{array}{lllllll}
c_{10} Z(G \otimes D) & Z K & 0 & c_{20} Z(G \otimes A) & 0 & Z
\end{array}\right],
\end{aligned}
$$

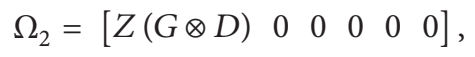

$$
\begin{aligned}
& \Omega_{2}=\left[\begin{array}{llllll}
0 & 0 & 0 & Z(G \otimes A) & 0 & 0
\end{array}\right], \\
& \widehat{\Omega}_{1}=\left[c_{10}(Z+p U)(G \otimes D) \quad(Z+p U) K \quad 0\right. \\
& \left.\times c_{20}(Z+p U)(G \otimes A) \quad 0 \quad(Z+p U)\right],
\end{aligned}
$$

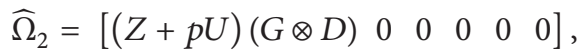

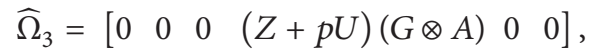

$$
\begin{aligned}
& X_{1}=\operatorname{diag}\{-Z,-Z,-Z\} \text {, } \\
& X_{2}=\operatorname{diag}\{-Z-p U,-Z-p U,-Z-p U\} \text {, } \\
& Z=p^{2} Z_{1}+h^{2} Z_{2}+p^{2} W \\
& \bar{R}=\operatorname{diag}\{0, R, 0,0,0,0\} \text {. }
\end{aligned}
$$

Proof. Consider the following Lyapunov functional:

$$
V(t)=V_{1}(t)+V_{2}(t)+V_{3}(t)+V_{4}(t)
$$

where $t \in\left[t_{k}, t_{k+1}\right)$ and

$$
\begin{aligned}
V_{1}(t)= & e^{T}(t) P e(t)+\int_{t-p}^{t} e^{T}(s) Q_{1} e(s) d s \\
& +\int_{t-h}^{t} e^{T}(s) Q_{2} e(s) d s \\
& +\int_{t-\tau(t)}^{t} e^{T}(s) Q_{3} e(s) d s, \\
V_{2}(t)= & \int_{-p}^{0} \int_{t+\theta}^{t} \dot{e}^{T}(s) Z_{1} \dot{e}(s) d s d \theta \\
& +h \int_{-h}^{0} \int_{t+\theta}^{t} \dot{e}^{T}(s) Z_{2} \dot{e}(s) d s d \theta, \\
V_{3}(t)= & \left(p-\left(t-t_{k}\right)\right) \int_{t_{k}}^{t} \dot{e}^{T}(s) U \dot{e}(s) d s \\
& +\left(p-\left(t-t_{k}\right)\right)\left(t-t_{k}\right) e^{T}\left(t_{k}\right) \operatorname{Re}\left(t_{k}\right),
\end{aligned}
$$

$$
\begin{aligned}
V_{4}(t)= & p^{2} \int_{t_{k}}^{t} \dot{e}^{T}(s) W \dot{e}(s) d s \\
& -\frac{\pi^{2}}{4} \int_{t_{k}}^{t}\left[x(s)-x\left(t_{k}\right)\right]^{T} W\left[x(s)-x\left(t_{k}\right)\right] d s .
\end{aligned}
$$

It is clear that at any $t>0$ except the sampling instants $t_{k}, V_{3}(t)$ is continuous and nonnegative, and right after the jump instants $t_{k}, V_{3}(t)$ becomes zero; that is, $V_{3}\left(t_{k}^{-}\right) \geq 0, V_{3}\left(t_{k}^{+}\right)=0$. According to Lemma 7 , we can easily find that $V_{4}(t) \geq 0$ and $V_{4}(t)$ vanishes at $t=t_{k}$. Thus, we have $V\left(t_{k}^{-}\right) \geq V\left(t_{k}^{+}\right)$.

Define the infinitesimal operator $L$ of $V(t)$ as follows:

$$
L V(t)=\lim _{\Delta \rightarrow 0+} \Delta^{-1}[E\{V(t+\Delta) \mid e(t)\}-V(t)]
$$

Taking the derivative of (16) along the solution of system (7) for $\forall t \in\left[t_{k}, t_{k+1}\right)$, it yields

$$
\begin{aligned}
& L V_{1}(t) \leq 2 e^{T}(t) \\
& \times P\left(\bar{g}(e(t))+c_{10}(G \otimes D) e(t)\right. \\
& +c_{20}(G \otimes A) e(t-\tau(t)) \\
& \left.+K e\left(t_{k}\right)\right)+e^{T}(t) Q_{1} e(t) \\
& -e^{T}(t-p) Q_{1} e(t-p) \\
& +e^{T}(t) Q_{2} e(t)-e^{T}(t-h) Q_{2} e(t-h) \\
& +e^{T}(t) Q_{3} e(t) \\
& -(1-\mu) e^{T}(t-\tau(t)) Q_{3} e(t-\tau(t)), \\
& L V_{2}(t)=\dot{e}^{T}(t) Z \dot{e}(t)-\int_{t-p}^{t} \dot{e}^{T}(s) Z_{1} \dot{e}(s) d s \\
& -h \int_{t-h}^{t} \dot{e}^{T}(s) Z_{2} \dot{e}(s) d s, \\
& L V_{3}(t)=\left(p-\left(t-t_{k}\right)\right) \dot{e}^{T}(t) U \dot{e}(t) \\
& -\int_{t_{k}}^{t} \dot{e}^{T}(s) U \dot{e}(s) d s \\
& +\left(p-\left(t-t_{k}\right)\right) e^{T}\left(t_{k}\right) \operatorname{Re}\left(t_{k}\right) \\
& -\left(t-t_{k}\right) e^{T}\left(t_{k}\right) \operatorname{Re}\left(t_{k}\right), \\
& L V_{4}(t)=p^{2} \dot{e}^{T}(t) W \dot{e}(t) \\
& -\frac{\pi^{2}}{4}\left[x(t)-x\left(t_{k}\right)\right]^{T} W\left[x(t)-x\left(t_{k}\right)\right] .
\end{aligned}
$$


If (14) is satisfied, then by utilizing Lemma 6, we have

$$
\begin{aligned}
& -p \int_{t-p}^{t} \dot{e}^{T}(s) Z_{1} \dot{e}(s) d s \\
& \leq-\left[\begin{array}{c}
e(t)-e\left(t_{k}\right) \\
e\left(t_{k}\right)-e(t-p)
\end{array}\right]^{T}\left[\begin{array}{cc}
Z_{1} & S_{1} \\
* & Z_{1}
\end{array}\right] \\
& \times\left[\begin{array}{c}
e(t)-e\left(t_{k}\right) \\
e\left(t_{k}\right)-e(t-p)
\end{array}\right] \\
& -h \int_{t-h}^{t} \dot{e}^{T}(s) Z_{2} \dot{e}(s) d s \\
& \leq-\left[\begin{array}{c}
e(t)-e(t-\tau(t)) \\
e(t-\tau(t))-e(t-h)
\end{array}\right]^{T}\left[\begin{array}{cc}
Z_{2} & S_{2} \\
* & Z_{2}
\end{array}\right] \\
& \times\left[\begin{array}{c}
e(t)-e(t-\tau(t)) \\
e(t-\tau(t))-e(t-h)
\end{array}\right] .
\end{aligned}
$$

On the other hand, the following inequality is true for any matrix $N$ with appropriate dimensions:

$$
\begin{aligned}
& -\int_{t_{k}}^{t} \dot{e}^{T}(s) U \dot{e}(s) d s \\
& \quad \leq\left(t-t_{k}\right) \xi^{T}(t) N U^{-1} N^{T} \xi(t) \\
& \quad+2 \xi^{T}(t) N\left(e(t)-e\left(t_{k}\right)\right),
\end{aligned}
$$

where

$$
\begin{aligned}
& \xi(t) \\
& =\left[\begin{array}{lllllll}
(t) & e^{T}\left(t_{k}\right) & e^{T}(t-p) & e^{T}(t-\tau(t)) & e^{T}(t-h) & \bar{g}^{T}(e(t))
\end{array}\right]^{T} .
\end{aligned}
$$

Let $\Omega=\Omega_{1}+\left(c_{1}(t)-c_{10}\right) \Omega_{2}+\left(c_{2}(t)-c_{20}\right) \Omega_{3}$. Because $c_{1}(t)$ and $c_{2}(t)$ are mutually independent random variables, it can be obtained from (7) that

$$
\begin{aligned}
& E\left\{\dot{e}^{T}(t) Z \dot{e}(t)\right\}=E\left\{\xi^{T}(t) \Omega^{T} Z \Omega \xi(t)\right\}=\xi^{T}(t) \bar{\Omega}_{1} \xi(t), \\
& E\left\{\dot{e}^{T}(t) U \dot{e}(t)\right\}=E\left\{\xi^{T}(t) \Omega^{T} U \Omega \xi(t)\right\}=\xi^{T}(t) \bar{\Omega}_{2} \xi(t),
\end{aligned}
$$

where $\bar{\Omega}_{1}=\Omega_{1}^{T} Z \Omega_{1}+\delta_{1}^{2} \Omega_{2}^{T} Z \Omega_{2}+\delta_{2}^{2} \Omega_{3}^{T} Z \Omega_{3}, \bar{\Omega}_{2}=$ $\Omega_{1}^{T} Z_{3} \Omega_{1}+\delta_{1}^{2} \Omega_{2}^{T} Z_{3} \Omega_{2}+\delta_{2}^{2} \Omega_{3}^{T} Z_{3} \Omega_{3}$. have

In addition, based on Assumption 3, for any $\varepsilon>0$, we

$$
y(t)=\varepsilon\left[\begin{array}{c}
e(t) \\
\bar{g}(e(t))
\end{array}\right]^{T}\left[\begin{array}{cc}
\bar{U} & \bar{V} \\
* & I
\end{array}\right]\left[\begin{array}{c}
e(t) \\
\bar{g}(e(t))
\end{array}\right] \leq 0 .
$$

Combining (18)-(24) and taking mathematical exceptions on both sides of (16) give that

$$
E\{L V(t)\} \leq \xi^{T}(t) \Phi \xi(t),
$$

where $\Phi=\Sigma+\bar{\Omega}_{1}+\left(p-\left(t-t_{k}\right)\right)\left(\bar{\Omega}_{2}+\bar{R}\right)+\left(t-t_{k}\right)\left(N U^{-1} N^{T}-\bar{R}\right)$.
Noting that $\Phi$ is a convex combination of $t-t_{k}$ and $p-$ $\left(t-t_{k}\right)$, so $\Phi<0$ if and only if

$$
\begin{gathered}
\Sigma+\bar{\Omega}_{1}+p N U^{-1} N^{T}-p \bar{R}<0, \\
\Sigma+\bar{\Omega}_{1}+p\left(\bar{\Omega}_{2}+\bar{R}\right)<0 .
\end{gathered}
$$

From the Schur complement, (12) and (13) can ensure $\Phi<$ 0 . This means that $E\{L V(t)\} \leq-\rho\|e(t)\|^{2}$ for a sufficiently small $\rho>0$. We can conclude that system (7) is asymptotically stable in the mean square sense. This completes the proof.

Remark 9. Inspired by $[20,22]$, the characteristic of sampling instants has been considered in the construction of the Lyapunov functional. The discontinuous terms $V_{3}(t)$ and $V_{4}(t)$ can make full use of the sawtooth structure characteristic of sampling instants and play the key role in the reduction of conservatism. In the process of taking the derivative of $V(t)$, reciprocally convex approach and convex combination technique were employed, which were beneficial to lead less conservativeness. Moreover, the derived synchronization criterion is formulated in terms of LMIs that can be easily verified by using available software.

Next, we will consider how to design the desired sampleddata controllers. Based on Theorem 8, a set of sampled-data controllers are presented as follows.

Theorem 10. Under Assumptions 3-4, the complex dynamical networks (1) with random coupling strength is globally asymptotically synchronized in mean square by the sampled-data controllers (5) if there exist matrices $P=\operatorname{diag}\left\{P_{1}, P_{2}, \ldots, P_{N}\right\}>$ $0, Q_{1}>0, Q_{2}>0, Q_{3}>0, Z_{1}>0, Z_{2}>0, U>$ $0, R>0, W>0, S_{1}, S_{2}, N, X=\operatorname{diag}\left\{X_{1}, X_{2}, \ldots, X_{N}\right\}$, and a scalar $\varepsilon>0$ such that (14) and the following LMIs are satisfied:

$$
\begin{gathered}
{\left[\begin{array}{ccc}
\widehat{\Sigma}-p \bar{R} & \Upsilon & p N \\
* & -\widehat{X}_{1} & 0 \\
* & * & -p U
\end{array}\right]<0,} \\
{\left[\begin{array}{cc}
\widehat{\Sigma}+p \bar{R} & \Upsilon \\
* & -\widehat{X}_{2}
\end{array}\right]<0,}
\end{gathered}
$$

where

$$
\begin{gathered}
\widehat{X}_{1}=\operatorname{diag}\{-2 P+Z,-2 P+Z,-2 P+Z\}, \\
\widehat{X}_{2}=\operatorname{diag}\{-2 P+Z+p U,-2 P \\
+Z+p U,-2 P+Z+p U\}, \\
\Upsilon=\left[\begin{array}{llllll}
\Upsilon_{1}^{T} & \delta_{1} \Upsilon_{2}^{T} & \delta_{2} \Upsilon_{3}^{T}
\end{array}\right], \\
\Upsilon_{1}=\left[\begin{array}{lllllll}
c_{10} P(G \otimes D) & X & 0 & c_{20} P(G \otimes A) & 0 & P
\end{array}\right], \\
\Upsilon_{2}=\left[\begin{array}{llllll}
P(G \otimes D) & 0 & 0 & 0 & 0 & 0
\end{array}\right],
\end{gathered}
$$




$$
\begin{gathered}
\Upsilon_{3}=\left[\begin{array}{llllll}
0 & 0 & 0 & P(G \otimes A) & 0 & 0
\end{array}\right], \\
\widehat{\Sigma}=\left[\begin{array}{cccccc}
\Sigma_{11} & \widehat{\Sigma}_{12} & \Sigma_{13} & \Sigma_{14} & \Sigma_{15} & \Sigma_{16} \\
* & \Sigma_{22} & \Sigma_{23} & -N_{4} & -N_{5} & -N_{6} \\
* & * & \Sigma_{33} & 0 & 0 & 0 \\
* & * & * & \Sigma_{44} & \Sigma_{45} & 0 \\
* & * & * & * & \Sigma_{55} & 0 \\
* & * & * & * & * & -\varepsilon I
\end{array}\right], \\
\widehat{\Sigma}_{12}=X+\frac{1}{4} \pi^{2} W+Z_{1}-S_{1}-N_{1}^{T}+N_{2},
\end{gathered}
$$

and the other terms follow the same definitions as those in Theorem 8. Moreover, the desired controllers gain matrices are given by

$$
K_{i}=P_{i}^{-1} X_{i}, \quad i=1,2, \ldots, N .
$$

Proof. Define matrices $X=P K, J_{1}=$ $\operatorname{diag}\left\{I, I, I, I, I, I, I, P Z^{-1}, P Z^{-1}, P Z^{-1}\right\}, \quad$ and $J_{2}=$ $\operatorname{diag}\left\{I, I, I, I, I, I, I, P(Z+p U)^{-1}, P(Z+p U)^{-1}, P(Z+p U)^{-1}\right\}$. Note that $-P Z^{-1} P \leq-2 P+Z$ and $-P(Z+p U)^{-1} P \leq$ $-2 P+Z+p U$ are true for $Z>0$ and $U>0$. Then, performing a congruence transformation of $J_{1}$ to (12) and performing a congruence transformation of $J_{2}$ to (13), respectively, and considering the relation $X=P K$, we can obtain that if (27) holds, then (12) and (13) hold. This completes the proof.

If $c_{1}(t)=c_{10}$ and $c_{2}(t)=c_{20}$, the random coupling strengths reduce to constant, and the error system (7) can be rewritten as following simple form:

$$
\begin{array}{r}
\dot{e}(t)=\bar{g}(e(t))+c_{10}(G \otimes D) e(t) \\
+c_{20}(G \otimes A) e(t-\tau(t))+K e\left(t_{k}\right), \\
\quad i=1,2, \ldots, N .
\end{array}
$$

Based on Theorems 8 and 10 , by eliminating $\delta_{1}$ and $\delta_{2}$, we can easily get the following results.

Corollary 11. Under Assumption 3, for given controller gain matrices $K_{i}$, the error system (30) with sampled-data controllers $K_{i}$ can achieve synchronization, if there exist matrices $P>0, Q_{1}>0, Q_{2}>0, Q_{3}>0, Z_{1}>0, Z_{2}>$ $0, U>0, R>0, W>0, S_{1}, S_{2}, N$, and a scalar $\varepsilon>0$ such that (10) and the following LMIs are satisfied:

$$
\begin{aligned}
& {\left[\begin{array}{ccc}
\Sigma-p \bar{R} & \Omega_{1}^{T} & p N \\
* & -Z & 0 \\
* & * & -p U
\end{array}\right]<0,} \\
& {\left[\begin{array}{cc}
\Sigma+p \bar{R} & \widehat{\Omega}_{1}^{T} \\
* & -Z-p U
\end{array}\right]<0,}
\end{aligned}
$$

where the other terms follow the same definitions as those in Theorem 8 .

Corollary 12. Under the Assumption 3, the complex dynamical network (1) with random coupling strengths is globally asymptotically synchronized in mean square by the sampled-data controllers (5) if there exist matrices $P=$ $\operatorname{diag}\left\{P_{1}, P_{2}, \ldots, P_{N}\right\}>0, Q_{1}>0, Q_{2}>0, Q_{3}>0, Z_{1}>$ $0, Z_{2}>0, U>0, R>0, W>0, S_{1}, S_{2}, N, X=$ $\operatorname{diag}\left\{X_{1}, X_{2}, \ldots, X_{N}\right\}$, and a scalar $\varepsilon>0$ such that (10) and the following LMIs are satisfied:

$$
\begin{aligned}
& {\left[\begin{array}{ccc}
\widehat{\Sigma}-p \bar{R} & \Upsilon_{1}^{T} & p N \\
* & -2 P+Z & 0 \\
* & * & -p U
\end{array}\right]<0,} \\
& {\left[\begin{array}{cc}
\widehat{\Sigma}+p \bar{R} & \Upsilon_{1} \\
* & -2 P+Z+p U
\end{array}\right]<0,}
\end{aligned}
$$

where the other terms follow the same definitions as those in Theorem 10.

Remark 13. Since the characteristic of sampled-data control system is fully considered, the conservatism of Corollary 12 is much less than those not taking delay characteristic into account $[27,28]$, which will be verified by numerical example in next section.

Remark 14. It is worth pointing out that the main result here can be extended to some more general complex dynamical networks with probabilistic time-varying coupling delay [18] or distributed coupling delay. Owing to the space limit, it is omitted here.

\section{Numerical Examples}

In this section, two numerical examples are given to show the validity of the proposed results.

Example 1. Consider complex network model (1) with three nodes. The out-coupling matrix is assumed to be $G=$ $\left(G_{i j}\right)_{N \times N}$ with

$$
G=\left[\begin{array}{ccc}
-1 & 0 & 1 \\
0 & -1 & 1 \\
1 & 1 & -2
\end{array}\right]
$$

The time-varying coupling delay is chosen as $\tau(t)=$ $0.2+0.05 \sin (10 t)$. A straight-forward calculation gives $h=$ 0.25 and $\mu=0.5$. The nonlinear function $f$ is taken as

$$
f\left(x_{i}(t)\right)=\left[\begin{array}{c}
-0.5 x_{i 1}+\tanh \left(0.2 x_{i 2}\right)+0.2 x_{i 2} \\
0.95 x_{i 2}-\tanh \left(0.75 x_{i 2}\right)
\end{array}\right] .
$$

It can be found that $f$ satisfies (2) with

$$
U=\left[\begin{array}{cc}
-0.5 & 0.2 \\
0 & 0.95
\end{array}\right], \quad V=\left[\begin{array}{cc}
-0.3 & 0.2 \\
0 & 0.2
\end{array}\right]
$$

The inner-coupling matrices are given as $D=0$ and

$$
A=\left[\begin{array}{ll}
1 & 0 \\
0 & 1
\end{array}\right]
$$

Let the coupling strength $c_{2}(t)$ be a constant; that is, $c_{2}(t)=c$. For different $c$, Table 1 lists the maximum sampling interval 
TABLE 1: Maximum sampling interval $p$ for different coupling strength $c$.

\begin{tabular}{lccc}
\hline & \multicolumn{1}{c}{$c$} & \\
& 0.5 & & 0.75 \\
\hline$[27]$ & 0.5409 & 0.1653 \\
{$[28]$} & 0.5573 & 0.2277 \\
{$[29]$} & 0.9016 & 0.8957 \\
Corollary 12 & 0.9795 & 0.9121 \\
\hline
\end{tabular}

$p$ obtained by Corollary 12 and [27-29]. From this table, we can see that our result has less conservatism than the existing ones.

Furthermore, choosing $c=0.5$ and applying MATLAB LMI toolbox to solve the LMIs in Corollary 12, the gain matrices of the desired controllers can be obtained as follows:

$$
\begin{aligned}
& K_{1}=\left[\begin{array}{ll}
-0.6578 & -0.0978 \\
-0.0172 & -1.2316
\end{array}\right], \\
& K_{2}=\left[\begin{array}{ll}
-0.6578 & -0.0978 \\
-0.0172 & -1.2316
\end{array}\right], \\
& K_{3}=\left[\begin{array}{ll}
-0.4543 & -0.1223 \\
-0.0185 & -1.1988
\end{array}\right] .
\end{aligned}
$$

In the numerical simulation, assume that the initial values are $x_{1}(0)=\left[\begin{array}{ll}3 & 5\end{array}\right]^{T}, x_{2}(0)=\left[\begin{array}{ll}-2 & -1\end{array}\right]^{T}, x_{3}(0)=$ $\left[\begin{array}{ll}2 & 1\end{array}\right]^{T}$, and $s(0)=\left[\begin{array}{ll}-5 & 3\end{array}\right]^{T}$. The state trajectories of the synchronization error and the control inputs $u_{i}(t)$ are given in Figures 1 and 2, respectively. Clearly, the synchronization errors are globally asymptotically stable in mean square under the proposed sampled-data scheme.

Example 2. The isolated node of the dynamical networks and the coupling delay are the same as Example 1. The innercoupling matrices are given as

$$
D=A=\left[\begin{array}{cc}
0.1 & 0 \\
0 & 0.1
\end{array}\right]
$$

and the outer-coupling matrix

$$
G=\left[\begin{array}{cccccc}
-3 & 1 & 0 & 2 & 0 & 0 \\
0 & -2 & 0 & 1 & 1 & 0 \\
1 & 0 & -3 & 1 & 0 & 1 \\
0 & 2 & 0 & -2 & 0 & 0 \\
1 & 1 & 1 & 1 & -4 & 0 \\
1 & 1 & 0 & 0 & 1 & -3
\end{array}\right]
$$

We assume that $c_{1}(t)$ and $c_{2}(t)$ are two mutually independent random variables satisfying normal distribution with $c_{10}=5, c_{20}=1, \delta_{1}=0.5$, and $\delta_{2}=0.15$. According to the property of normal distribution, almost all the values of $c_{i}(t)$ satisfy $c_{1}(t) \in\left(c_{i 0}-3 \delta_{i}, c_{i 0}+3 \delta_{i}\right)$; that is $c_{1}(t) \in$ $(3.5,6.5)$ and $c_{2}(t) \in(1.55,2.45)$. Figures 3 and 4 depict the random coupling strengths $c_{1}(t)$ and $c_{2}(t)$, respectively.

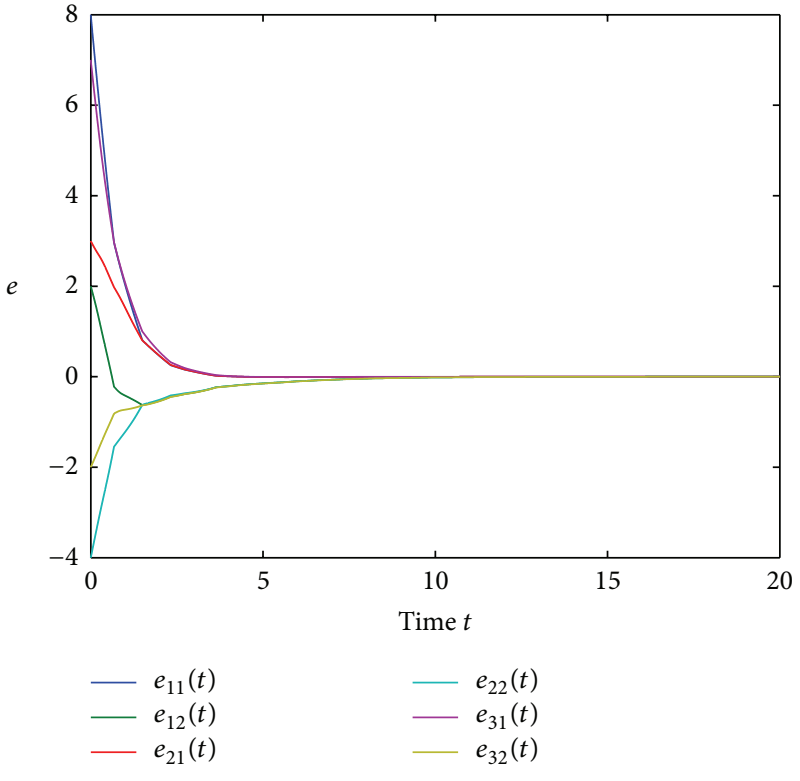

FIGURE 1: Synchronization error states.

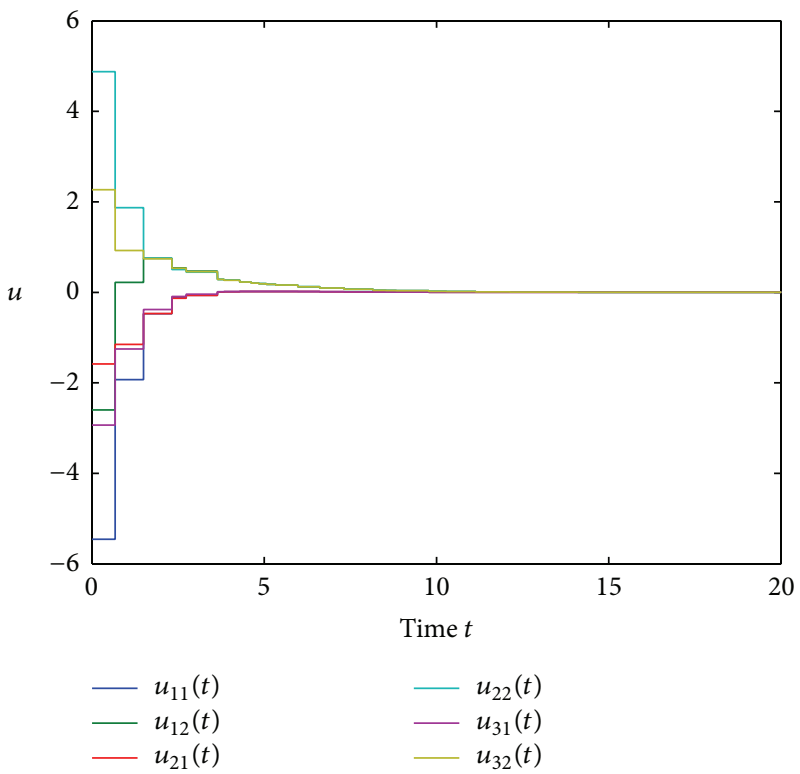

FIGURE 2: Sampled-data control inputs.

Let $p=0.05$; based on Theorem 10, we can get the corresponding sampled-data controller gain matrices

$$
\begin{aligned}
& K_{1}=\left[\begin{array}{ll}
-0.1314 & -0.1212 \\
-0.0709 & -1.1841
\end{array}\right], \\
& K_{2}=\left[\begin{array}{ll}
-0.4319 & -0.1243 \\
-0.0753 & -1.3982
\end{array}\right], \\
& K_{3}=\left[\begin{array}{cc}
0.1045 & -0.1144 \\
-0.0747 & -0.9176
\end{array}\right],
\end{aligned}
$$




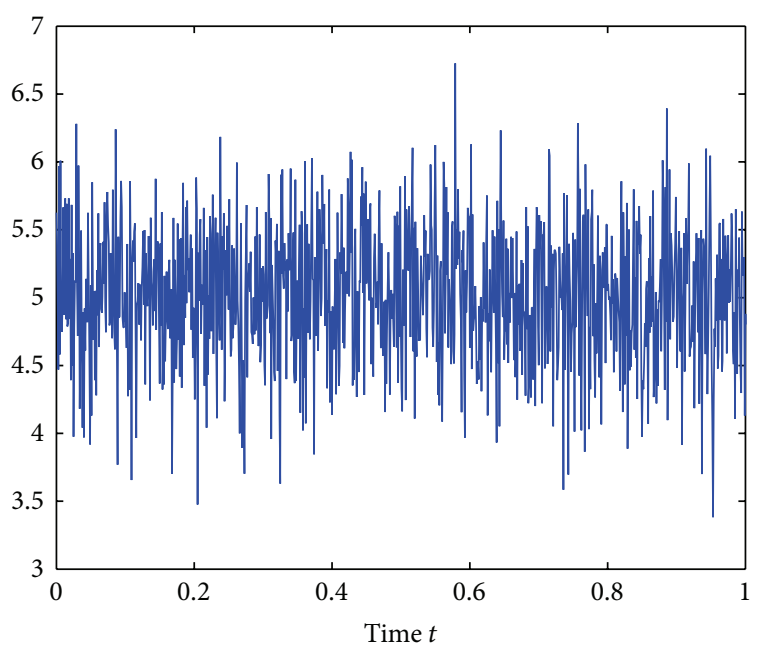

FIGURE 3: Random coupling strength $c_{1}(t)$.

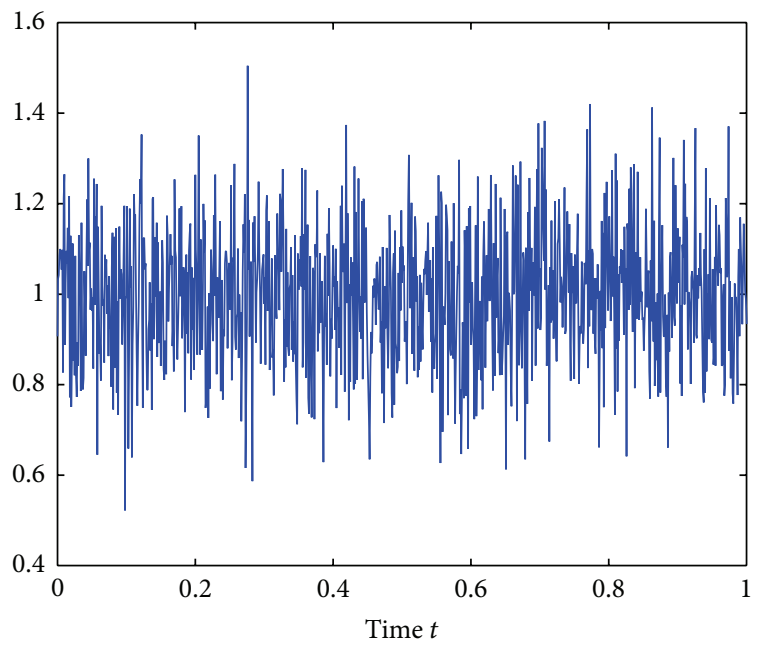

FIGURE 4: Random coupling strength $c_{2}(t)$.

$$
\begin{aligned}
& K_{4}=\left[\begin{array}{ll}
-0.5565 & -0.1190 \\
-0.0665 & -1.5593
\end{array}\right], \\
& K_{5}=\left[\begin{array}{cc}
0.5769 & -0.1184 \\
-0.0688 & -0.4136
\end{array}\right], \\
& K_{6}=\left[\begin{array}{cc}
0.1801 & -0.1154 \\
-0.0846 & -0.8069
\end{array}\right] .
\end{aligned}
$$

In the numerical simulation, assume that the initial values are $x_{1}(0)=\left[\begin{array}{ll}3 & 2\end{array}\right]^{T}, x_{2}(0)=\left[\begin{array}{ll}-1 & -3\end{array}\right]^{T}, x_{3}(0)=$ $\left[\begin{array}{ll}2 & 4\end{array}\right]^{T}, x_{4}(0)=\left[\begin{array}{ll}5 & -1\end{array}\right]^{T}, x_{5}(0)=\left[\begin{array}{ll}-4 & 3\end{array}\right]^{T}, x_{6}(0)=$ $\left[\begin{array}{ll}4 & 1\end{array}\right]^{T}$, and $s(0)=\left[\begin{array}{ll}3 & -4\end{array}\right]^{T}$. The state trajectories of the synchronization error and the control inputs $u_{i}(t)$ are given in Figures 5 and 6, respectively.

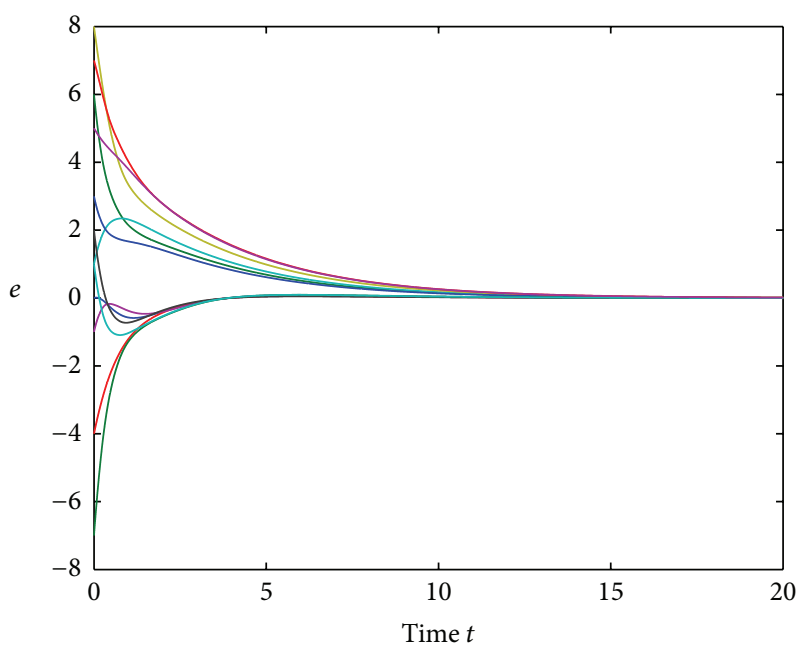

FIGURE 5: Synchronization error states.

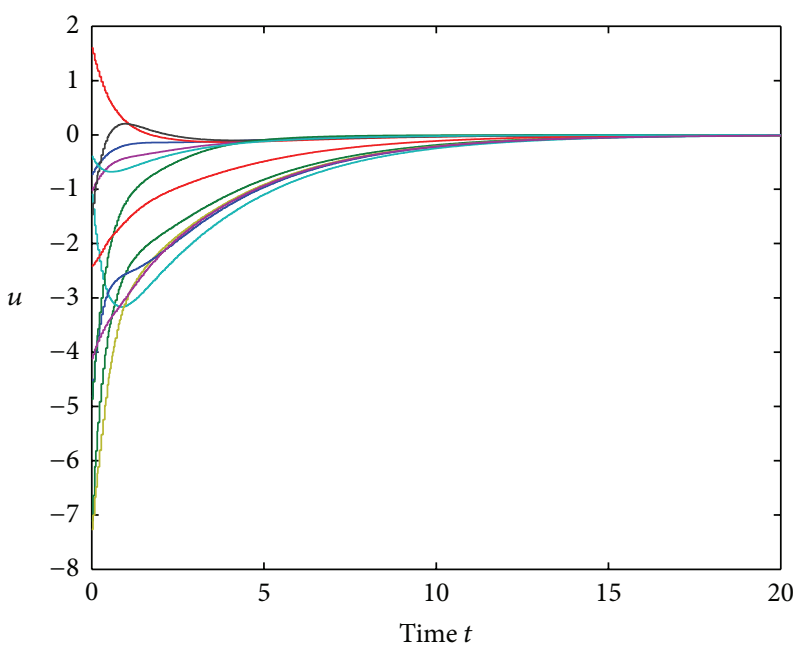

Figure 6: Sampled-data control inputs.

\section{Conclusions}

In this paper, the sampled-data synchronization problem has been considered for a kind of complex dynamical networks with time-varying coupling delay and random coupling strengths. The sampling period and random coupling strengths considered here are assumed to be time varying but bounded and to obey normal distribution, respectively. By capturing the characteristic of sampled-data system, a novel discontinuous Lyapunov functional is defined. By using reciprocally convex approach and convex combination technique, a mean square synchronization criterion is proposed based on LMIs. The corresponding desired sampleddata controllers are designed. Numerical examples show the effectiveness of the proposed result. 


\section{Conflict of Interests}

The authors declare that there is no conflict of interests regarding the publication of this paper.

\section{Acknowledgments}

The work is Supported by the National Natural Science Foundation of China (Grant nos. 61203049 and 61303020), the Doctoral Startup Foundation of Taiyuan University of Science and Technology (Grant no. 20112010), and Shanxi Education Department Foundation (Grant no. 20121068).

\section{References}

[1] S. H. Strogatz, "Exploring complex networks," Nature, vol. 410, no. 6825 , pp. $268-276,2001$.

[2] R. Albert and A.-L. Barabási, "Statistical mechanics of complex networks," Reviews of Modern Physics, vol. 74, no. 1, pp. 47-97, 2002.

[3] X. F. Wang and G. R. Chen, "Complex networks: small-world, scale-free and beyond," IEEE Circuits and Systems Magazine, vol. 3, no. 1, pp. 6-20, 2003.

[4] X. F. Wang and G. R. Chen, "Synchronization in scale-free dynamical networks: robustness and fragility," IEEE Transactions on Circuits and Systems. I. Fundamental Theory and Applications, vol. 49, no. 1, pp. 54-62, 2002.

[5] Y. Wang, Z. D. Wang, and J. L. Liang, "A delay fractioning approach to global synchronization of delayed complex networks with stochastic disturbances," Physics Letters A: General, Atomic and Solid State Physics, vol. 372, no. 39, pp. 6066-6073, 2008.

[6] J. Zhou, J.-A. Lu, and J. Lü, "Adaptive synchronization of an uncertain complex dynamical network," Institute of Electrical and Electronics Engineers. Transactions on Automatic Control, vol. 51, no. 4, pp. 652-656, 2006.

[7] J. Q. Lu, W. D. C. Ho, and J. D. Cao, "A unified synchronization criterion for impulsive dynamical networks," Automatica, vol. 46, no. 7, pp. 1215-1221, 2010.

[8] W. W. Yu, G. R. Chen, and J. H. Lü, "On pinning synchronization of complex dynamical networks," Automatica, vol. 45, no. 2, pp. 429-435, 2009.

[9] Q. Song and J. D. Cao, "On pinning synchronization of directed and undirected complex dynamical networks," IEEE Transactions on Circuits and Systems. I. Regular Papers, vol. 57, no. 3, pp. 672-680, 2010.

[10] J. Lu and W. D. C. Ho, "Globally exponential synchronization and synchronizability for general dynamical networks," IEEE Transactions on Systems, Man, and Cybernetics B: Cybernetics, vol. 40, no. 2, pp. 350-361, 2010.

[11] J. Yao, Z.-H. Guan, and D. J. Hill, "Passivity-based control and synchronization of general complex dynamical networks," Automatica, vol. 45, no. 9, pp. 2107-2113, 2009.

[12] M. J. Park, O. M. Kwon, J. H. Park, S. M. Lee, and E. J. Cha, "Synchronization criteria for coupled stochastic neural networks with time-varying delays and leakage delay," Journal of the Franklin Institute. Engineering and Applied Mathematics, vol. 349, no. 5, pp. 1699-1720, 2012.

[13] Y. H. Xu, W. N. Zhou, J. A. Fang, W. Sun, and L. Pan, “Topology identification and adaptive synchronization of uncertain complex networks with non-derivative and derivative coupling,"
Journal of the Franklin Institute. Engineering and Applied Mathematics, vol. 347, no. 8, pp. 1566-1576, 2010.

[14] C. W. Wu, "Synchronization and convergence of linear dynamics in random directed networks," Institute of Electrical and Electronics Engineers. Transactions on Automatic Control, vol. 51, no. 7, pp. 1207-1210, 2006.

[15] T. H. Lee, J. H. Park, D. H. Ji, O. M. Kwon, and S. M. Lee, "Guaranteed cost synchronization of a complex dynamical network via dynamic feedback control," Applied Mathematics and Computation, vol. 218, no. 11, pp. 6469-6481, 2012.

[16] J.-L. Wang and H.-N. Wu, "Local and global exponential output synchronization of complex delayed dynamical networks," Nonlinear Dynamics. An International Journal of Nonlinear Dynamics and Chaos in Engineering Systems, vol. 67, no. 1, pp. 497-504, 2012.

[17] X. S. Yang, J. D. Cao, and J. Q. Lu, "Synchronization of Markovian coupled neural networks with nonidentical modedelays and random coupling strengths," IEEE Transactions on Neural Networks and Learning Systems, vol. 23, no. 1, pp. 60-71, 2012.

[18] X. S. Yang, J. D. Cao, and J. Q. Lu, "Synchronization of coupled neural networks with random coupling strengths and mixed probabilistic time-varying delays," International Journal of Robust and Nonlinear Control, vol. 23, no. 18, pp. 2060-2081, 2013.

[19] E. Fridman, A. Seuret, and J.-P. Richard, "Robust sampleddata stabilization of linear systems: an input delay approach," Automatica, vol. 40, no. 8, pp. 1441-1446, 2004.

[20] E. Fridman, "A refined input delay approach to sampled-data control," Automatica, vol. 46, no. 2, pp. 421-427, 2010.

[21] B. H. K. Lam, "Output-feedback sampled-data polynomial controller for nonlinear systems," Automatica, vol. 47, no. 11, pp. 2457-2461, 2011.

[22] K. Liu and E. Fridman, "Wirtinger's inequality and Lyapunovbased sampled-data stabilization," Automatica, vol. 48, no. 1, pp. 102-108, 2012.

[23] J. G. Lu and D. J. Hill, "Global asymptotical synchronization of chaotic Lur'e systems using sampled data: a linear matrix inequality approach," IEEE Transactions on Circuits and Systems II: Express Briefs, vol. 55, no. 6, pp. 586-590, 2008.

[24] Z. G. Wu, P. Shi, H. Y. Su, and J. Chu, "Exponential synchronization of neural networks with discrete and distributed delays under time-varying sampling," IEEE Transactions on Neural Networks and Learning Systems, vol. 23, no. 9, pp. 1368-1376, 2012.

[25] Z. G. Wu, P. Shi, H. Y. Su, and J. Chu, "Stochastic synchronization of Markovian jump neural networks with time-varying delay using sampled-data," IEEE Transactions on Cybernetics, vol. 43, no. 6, pp. 1796-1806, 2013.

[26] C. K. Zhang, Y. He, and M. Wu, "Exponential synchronization of neural networks with time-varying mixed delays and sampled-data," Neurocomputing, vol. 74, no. 1-3, pp. 265-273, 2010.

[27] N. Li, Y. L. Zhang, J. W. Hu, and Z. Y. Nie, "Synchronization for general complex dynamical networks with sampled-data," Neurocomputing, vol. 74, no. 5, pp. 805-811, 2011.

[28] Z.-G. Wu, J. H. Park, H. Su, B. Song, and J. Chu, "Exponential synchronization for complex dynamical networks with sampled-data," Journal of the Franklin Institute. Engineering and Applied Mathematics, vol. 349, no. 9, pp. 2735-2749, 2012. 
[29] Z. G. Wu, P. Shi, H. Y. Su, and J. Chu, "Sampled-data exponential synchronization of complex dynamical networks with timevarying coupling," IEEE Transactions on Neural Networks and Learning Systems, vol. 24, pp. 1177-1187, 2013.

[30] P. Park, J. W. Ko, and C. Jeong, "Reciprocally convex approach to stability of systems with time-varying delays," Automatica, vol. 47, no. 1, pp. 235-238, 2011. 


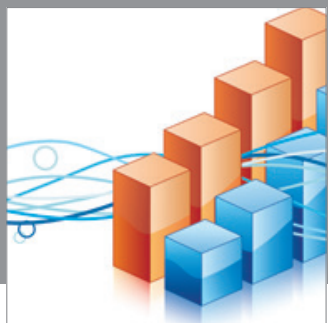

Advances in

Operations Research

mansans

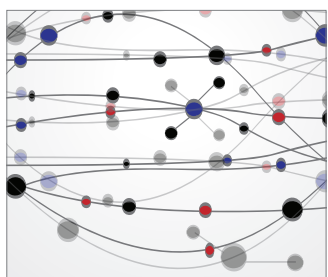

The Scientific World Journal
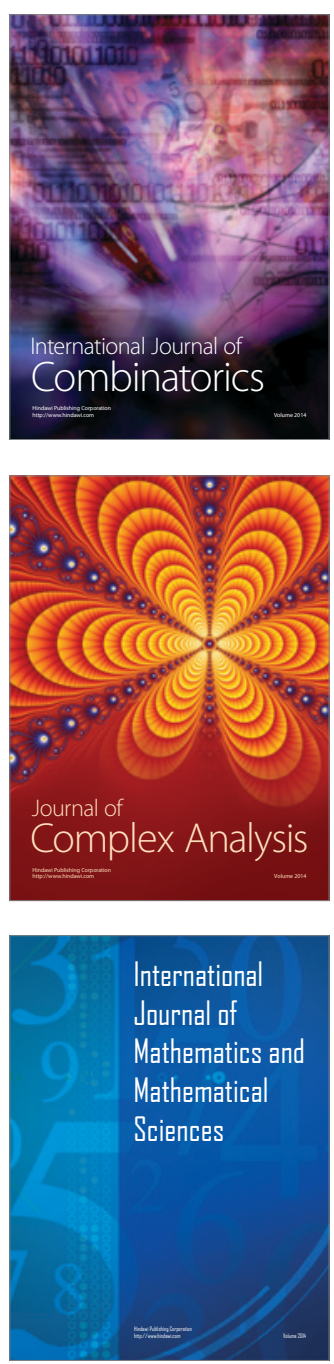
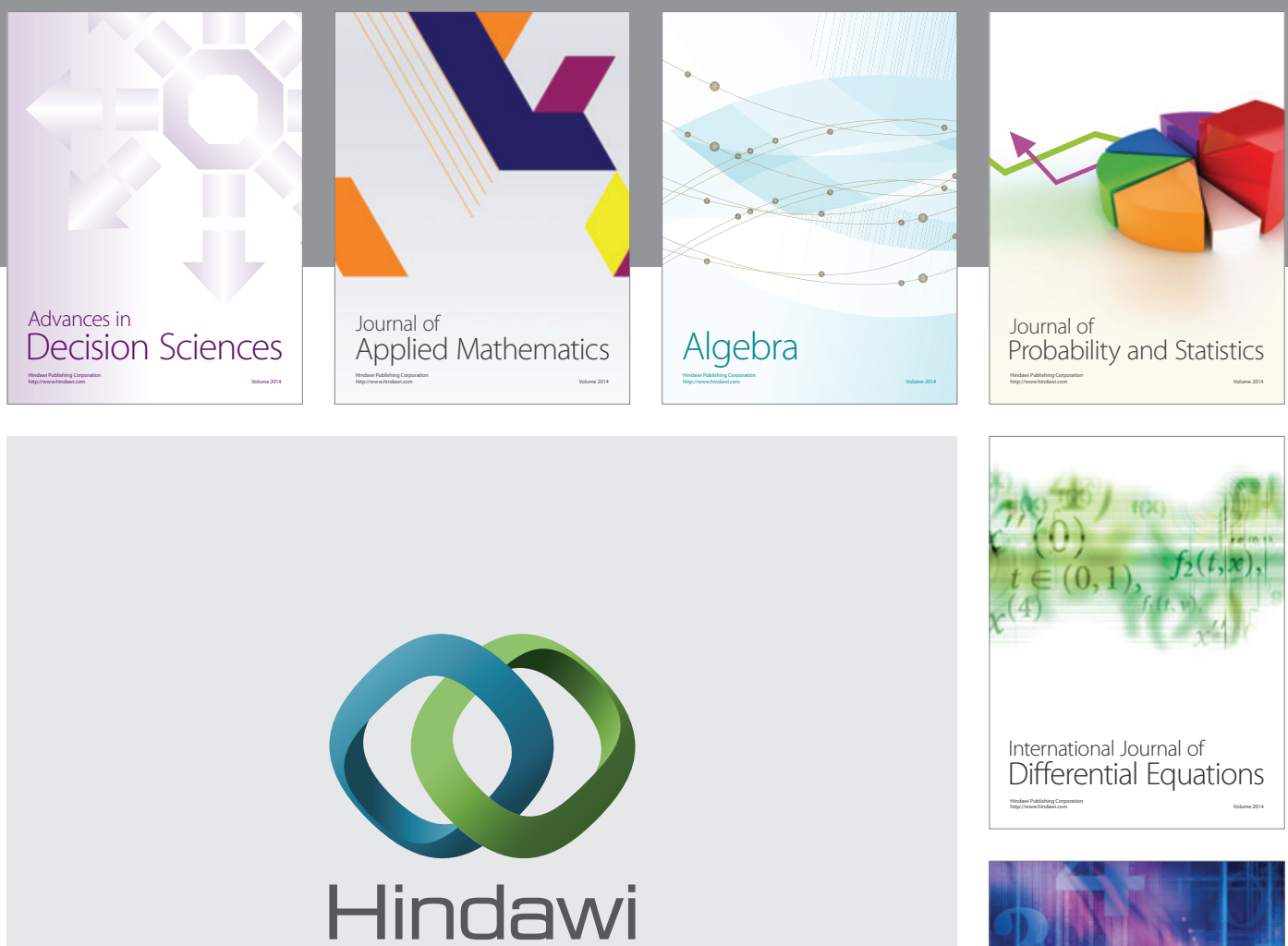

Submit your manuscripts at http://www.hindawi.com
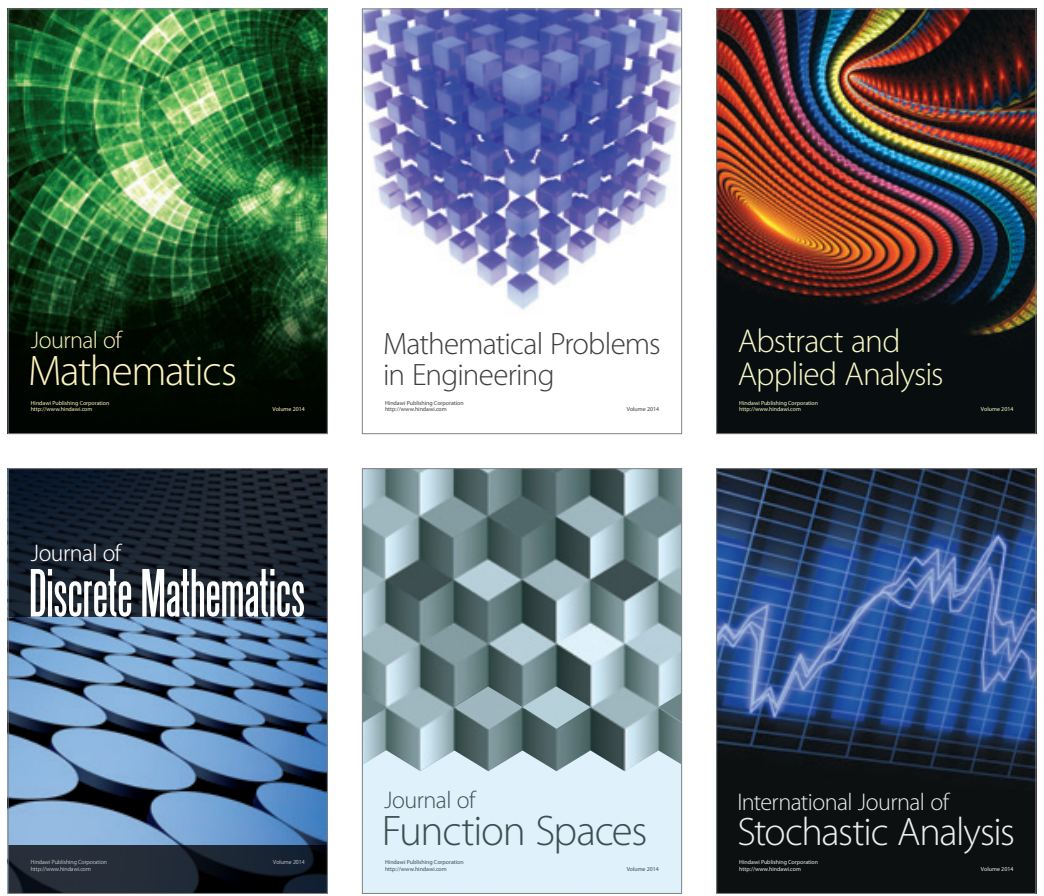

Journal of

Function Spaces

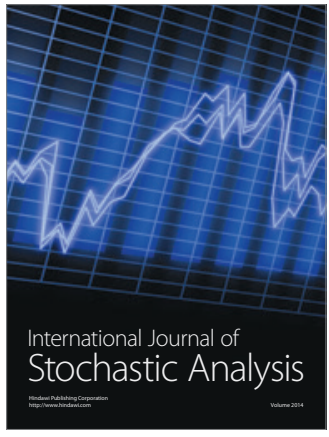

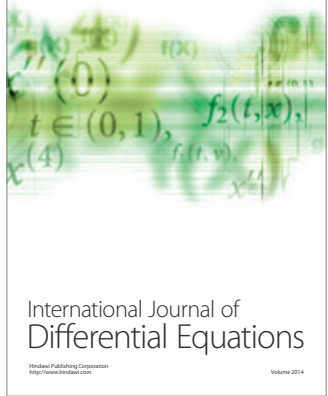
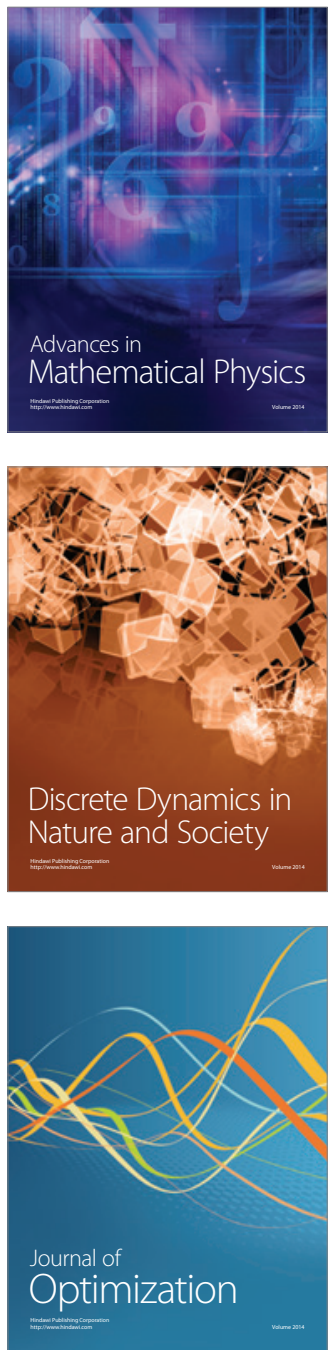